\title{
Ciliary metaplasia in a patient with antineutrophil cytoplasmic autoantibody-associated pauci-immune glomerulonephritis
}

\author{
Chang-Hoon Lee ${ }^{1}$, Jin Han $\mathrm{Lim}^{1}$, Kyoung Min Kim², Moon Hyang Park², and Kyung Pyo Kang ${ }^{1}$
}

\begin{abstract}
${ }^{1}$ Department of Internal Medicine, Research Institute of Clinical Medicine, ${ }^{2}$ Department of Pathology, Chonbuk National University Medical School, Jeonju; ${ }^{3}$ Department of Pathology, Konyang University Hospital, Daejeon, Korea
\end{abstract}

Received: September 6, 2017 Revised : September 8, 2017 Accepted: September 8, 2017

\section{Correspondence to}

Kyung Pyo Kang, M.D.

Tel: +82-63-250-2361

Fax: +82-63-254-1609

E-mail:kpkang@chonbuk.ac.kr
A 73-year-old man was admitted to the hospital due to generalized edema with renal dysfunction. Laboratory tests revealed a white blood cell count of $9,640 / \mathrm{mm}^{3}$, hemoglobin of $8.7 \mathrm{~g} / \mathrm{dL}$, platelets of $199,000 / \mathrm{mm}^{3}$, blood urea nitrogen of $71 \mathrm{mg} / \mathrm{dL}$, and serum creatinine of $4.25 \mathrm{mg} / \mathrm{dL}$. Urinalysis revealed $1+$ proteinuria and microscopic hematuria. The urinary protein/ creatinine ratio was $1,284 \mathrm{mg} / \mathrm{g}$. Serum myeloperoxidase-antineutrophil cytoplasmic autoantibody (p-ANCA) was positive (over 100.0 U/mL). We suspected clinically ANCA-associated pauci-immue glomerulonephritis and performed a renal biopsy. The renal biopsy specimen contained approximately 38 glomeruli, of which 14 were globally sclerotic. Approximately $29 \%$ of the glomeruli had a varying degree of segmental necrotizing and crescentic glomerulonephritis with sclerosis (Fig. 1A). The interstitium was expanded by diffuse lymphocytic infiltrates, focal edema and fibrosis associated with tubular injury and tubulitis (Fig. 1B). On electron microscopy, the tubules showed diminished apical microvilli along the proximal tubules, compatible with acute tubular injury and diffuse tubulointerstitial inflammation. A cluster of cilia was noted along the tubular cell apical surfaces (Fig. 1C). Under high magnification, the cilia had 9 pairs of peripheral microtubules with a central pair of microtubules; this was consistent with motile $(9+2)$ rather than primary cilia $(9+0)$ (Fig. 1D). The biopsy findings
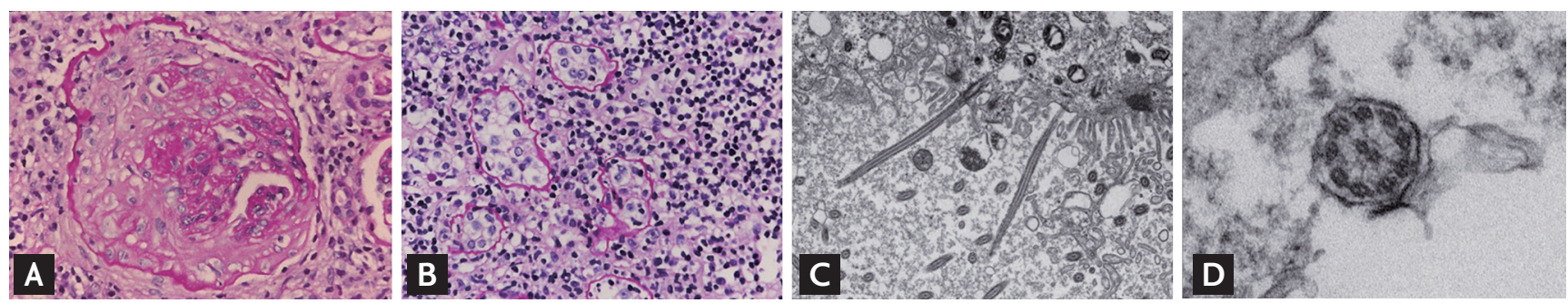

Figure 1. Histologic features of renal biopsy. (A) Light microscopy shows a large circumferential fibrocellular crescent surrounding segmentally sclerosing and collapsed glomerular tufts (PAS stain, $\times 400$ ). (B) The interstitium is expanded by interstitial inflammation mainly consisting of lymphocytes and some plasma cells. Lymphocytes that invade the tubular epithelium (tubulitis) accompanied by tubular degenerative change (PAS stain, $\times 400$ ). (C) Electron microscopy reveals clusters of cilia along the apical surface near diminished proximal tubular cells microvilli (original magnification, $\times 3,000)$. (D) Under higher magnification, the cilia exhibit 9 pairs of peripheral microtubules with a central pair of microtubules, consistent with motile cilia $(9+2)$ (original magnification, $\times 30,000)$. 
were interpreted as being consistent with ANCA-associated crescentic glomerulonephritis (mixed histologic class) associated with marked tubulointerstitial nephritis and ciliary metaplasia.

Cilia are highly conserved organelles that act as mechanosensors, chemosensors, and photosensors. In renal pathology processes such as crescentic glomerulonephritis, membranoproliferative glomerulonephritis, lupus nephritis, congenital nephrotic syndrome, amyloidosis, sarcoidosis, and acute tubular injury in renal transplant, clusters of ciliary metaplasia, both primary $(9+0)$ and motile $(9+2)$, have been found in injured tubules. This so-called "ciliary metaplasia" might be associated with changes to a more primitive or less-differentiated state and reparative processes. However, underlying mechanism of cilia metaplasia remains unclear. We need to further investigate the mechanisms in ciliary metaplasia after pathologic renal injury.

We had obtained written informed consent from the patient for the publication of this case and pathologic images.

\section{Conflict of interest}

No potential conflict of interest relevant to this article was reported. 